\title{
Civic Crowdfunding for Agents with Negative Valuations and Agents with Asymmetric Beliefs
}

\author{
Sankarshan Damle ${ }^{1 *}$, Moin Hussain Moti ${ }^{1}$, Praphul Chandra ${ }^{2}$ and Sujit Gujar ${ }^{1}$ \\ ${ }^{1}$ Machine Leaning Lab, International Institute of Information Technology, Hyderabad \\ ${ }^{2}$ KoineArth, Bangalore \\ \{sankarshan.damle,moin.moti\}@ research.iiit.ac.in, praphulcs@koinearth.com, sujit.gujar@iiit.ac.in
}

\begin{abstract}
In the last decade, civic crowdfunding has proved to be effective in generating funds for the provision of public projects. However, the existing literature deals only with citizen's with positive valuation and symmetric belief towards the project's provision. In this work, we present novel mechanisms which break these two barriers, i.e., mechanisms which incorporate negative valuation and asymmetric belief, independently. For negative valuation, we present a methodology for converting existing mechanisms to mechanisms that incorporate agents with negative valuations. Particularly, we adapt existing PPR and PPS mechanisms, to present novel PPRN and PPSN mechanisms which incentivize strategic agents to contribute to the project based on their true preferences. With respect to asymmetric belief, we propose a reward scheme Belief Based Reward (BBR) based on Robust Bayesian Truth Serum mechanism. With BBR, we propose a general mechanism for civic crowdfunding which incorporates asymmetric agents. We leverage PPR and PPS, to present PPRx and PPSx. We prove that in PPRx and PPSx, agents with greater belief towards the project's provision contribute more than agents with lesser belief. Further, we also show that contributions are such that the project is provisioned at equilibrium.
\end{abstract}

\section{Introduction}

Crowdfunding is a process of raising funds from a large pool of interested agents and is an active area of research [Alaei et al., 2016; Arieli et al., 2017; Chandra et al., 2017; Strausz, 2017; Shen et al., 2018]. The process, when applied for the provision of public projects, is called civic crowdfunding. In the last decade, civic crowdfunding has grown to be instrumental in providing a platform through which citizens can collectively finance social initiatives such as libraries, public parks, etc.

In the standard approach for civic crowdfunding, the social planner uses the voluntary contribution mechanism with a

\footnotetext{
${ }^{*}$ Contact Author
}

provision point, the provision point mechanism [Bagnoli and Lipman, 1989]. The social planner sets up a target amount, referred to as the provision point, to be raised. If the contributions by the agents exceed the provision point, social planner provisions the project; otherwise, returns the contributions. The mechanism, however, has been shown to have several inefficient equilibria [Bagnoli and Lipman, 1989; Brubaker, 1975; Schmidtz, 1991].

Provision Point mechanism with Refund bonus (PPR) by Zubrickas [2014] introduces an additional refund bonus to be paid to all the contributing agents (along with their contribution) in case the project is not provisioned. Chandra et $a l$. [2016] showed that in sequential setting, wherein the history of contributions is known to the agents, PPR collapses to a simultaneous-move game, among the contributing agents. Towards this, they proposed Provision Point mechanism with Securities (PPS) with refunds based on complex prediction markets, and showed that it induces a sequential game, in which the project is provisioned at equilibrium. Thus in this paper, for a sequential game, we focus on PPS while focusing on PPR for a simultaneous game.

Note that in all these mechanisms only those agents with positive valuations towards the project contribute to its provision. However, several agents may prefer the project to not be provisioned, i.e., their valuations may be negative for the project getting provisioned. For instance, consider the construction of a garbage dump yard in a locality. While the project may be welcomed by a number of agents, a certain set of agents may wish to relocate the project from its current location to another. In other words, these agents may not prefer the construction of the dump yard - in the locality proposed. In such a scenario, the construction of the dump yard (as well as the locality in which it is constructed) must depend on the majority's opinion of it. The civic crowdfunding literature does not address such negative valuations. If addressed, civic crowdfunding with agents with negative valuations can provide a natural way for preference aggregation.

We define an agent's information structure as consisting of its valuation and its belief towards the project's provision. Based on their valuation, we categorize these agents as follows: positive (negative) agents i.e., agents with positive (negative) valuations or positive (negative) preferences towards the project's provision. The mechanisms, mentioned above, also assume that apart from knowing the history of 
contributions, agents do not have any information regarding the provision of the project, i.e., every agent's belief is symmetric towards the project's provision.

Motivated to break these barriers on an agent's information structure in existing literature for civic crowdfunding, in this paper, we address these two limitations by (i) handling symmetric agents with negative preferences and (ii) handling positive agents with asymmetric belief towards the project's provision, independently. Relaxing both the assumptions in one mechanism is still illusive.

To incorporate civic crowdfunding for agents with negative valuations, we require mechanisms that integrate negative agents. For this, we set up two parallel markets, with two different targets - one for the provision, i.e., provision point and one against the provision, i.e., rejection point, for the project. The project is provisioned (not provisioned) if the provision (rejection) point is reached first. A strategic agent may choose to contribute in a market, against its preference. Thus, the challenge in such a setting remains to ingeniously design a refund scheme such that the agents are incentivized to contribute based on their preferences. For this, we propose a methodology through which existing mechanisms for positive preferences also allow for agents with negative preferences, such that agents contribute to the market based on their true preferences. In particular, we adapt existing PPR and PPS mechanisms to design PPRN and PPSN mechanisms. We prove that in these mechanisms at equilibrium, either the provision point or the rejection point holds.

Further, designing mechanisms for civic crowdfunding for agents with asymmetric beliefs is not trivial. For instance, a rational agent with significant belief towards the project's provision may choose to free-ride, as it believes that the project will be provisioned regardless of its contribution. Such asymmetric agents need to be further incentivized to contribute towards the project's provision. For this, we propose a novel reward scheme Belief Based Reward (BBR) that rewards an agent based on their belief towards the project's provision. We deploy a peer prediction mechanism for information aggregation of each agent's belief. With BBR, we propose a novel class of mechanisms for civic crowdfunding which incentivizes agents with asymmetric beliefs to contribute towards the provision, such that the project is provisioned at equilibrium. To the best of our knowledge, there is no prior work addressing negative valuations and asymmetric beliefs in civic crowdfunding literature.

\section{Preliminaries}

In this section, we present the required preliminaries. We begin by defining our crowdfunding model.

\subsection{Model}

For the civic crowdfunding of public project $P P$, the Project Maker (PM), sets up a market for its provision. The PM announces a provision point $h^{0}$ as the target to be reached until a deadline $T$. Let $\mathbb{A}=\{1, \ldots, n\}$ be the set of all contributing agents wherein each Agent $i$ has valuation $\theta_{i} \geq 0$ if $P P$ gets provisioned. The agents contribute $x=\left(x_{1}, \ldots, x_{n}\right)$ to the crowdfunding mechanism. Let $\vartheta=\sum_{i=1}^{i=n} \theta_{i}$; the total valuation of all the agents and $\mathcal{X}=\sum_{i=1}^{i=n} x_{i}$; the sum of the contributions. A project is provisioned if $\mathcal{X} \geq h^{0}$ at the end of deadline $T$ and not provisioned otherwise. Such mechanisms are referred to as provision point mechanisms. Note that $\theta_{i} \mathrm{~s}$ are private to the agents and provision point mechanisms are indirect mechanisms to aggregate these. This setup induces a game among the agents.

Let $\sigma=\left(\sigma_{1}, \ldots, \sigma_{n}\right)$ be the vector of strategy profile of every agent. Agent $i$ 's strategy consists of its contribution $x_{i}$ towards the project's provision along with other mechanism dependent parameters. We use the subscript $-i$ to represent vectors without Agent $i$. The utility for Agent $i$ with valuation $\theta_{i}$ for the project, when all the agents play the strategy profile $\sigma$ is $u_{i}\left(\sigma ; \theta_{i}\right)$.

We now define some game-theoretic definitions necessary for the analysis of the mechanisms presented in this paper.

Definition 1 (Nash Equilibrium (NE)). A strategy profile $\sigma^{*}=\left(\sigma_{1}^{*}, \ldots, \sigma_{n}^{*}\right)$ is said to be a Nash equilibrium $(N E)$ if for every Agent $i$, it maximizes the utility $u_{i}\left(\sigma^{*} ; \theta_{i}\right)$ i.e., $\forall i \in \mathbb{A}$,

$$
u_{i}\left(\sigma_{i}^{*}, \sigma_{-i}^{*} ; \theta_{i}\right) \geq u_{i}\left(\sigma_{i}, \sigma_{-i}^{*} ; \theta_{i}\right) \forall \sigma_{i}, \forall \theta_{i} .
$$

For crowdfunding in sequential setting, i.e., when the contributing agents arrive over time, the strategy profile of every Agent $i$ also constitutes the time $t_{i}$ at which it contributes to the mechanism. Let, $a_{i}$ be the time at which Agent $i$ arrives to the mechanism. Further, let $h_{t}^{0}$ denote the amount remaining for the project to be provisioned at time $t$. With this, we define,

Definition 2 (Sub-game Perfect Equilibrium (SPE)). A strategy profile $\sigma^{*}=\left(\sigma_{1}^{*}, \ldots, \sigma_{n}^{*}\right)$ is said to be a sub-game perfect equilibrium if for every Agent $i$, it maximizes the utility $u_{i}\left(\sigma_{i}^{*}, \sigma_{-i \mid H^{a_{i}}} ; \theta_{i}\right)$ i.e., $\forall i \in \mathbb{A}$,

$$
u_{i}\left(\sigma_{i}^{*}, \sigma_{-i \mid H^{a_{i}}}^{*} ; \theta_{i}\right) \geq u_{i}\left(\sigma_{i}, \sigma_{-i \mid H^{a_{i}}} ; \theta_{i}\right) \forall \sigma_{i}, \forall H^{t}, \forall \theta_{i} .
$$

Here, $H^{t}$ is the history of the game till time $t$, constituting the agents' arrivals and their contributions and $\sigma_{-i \mid H^{a_{i}}}^{*}$ indicates that the players who arrive after $a_{i}$ follow the strategy specified by $\sigma_{-i}^{*}$.

In the next subsection, we describe existing mechanisms in the literature for civic crowdfunding.

\subsection{Provision Point Mechanisms}

In this paper, we focus on the class of mechanisms for civic crowdfunding which require the project to aggregate a minimum level (Provision Point of the project) of funding before the PM can claim it. Several provision point mechanisms for civic crowdfunding have been proposed [Brubaker, 1975; Groves and Ledyard, 1977; Bagnoli and Lipman, 1989; Schmidtz, 1991; Tabarrok, 1998; Chen, 2008; Chandra et al., 2017; Damle et al., 2018]; but we focus on PPR [Zubrickas, 2014] and PPS [Chandra et al., 2016] mechanisms.

\section{Provision Point Mechanism with Refund (PPR)}

To counter the problem of free-riding, PPR offers a refund bonus to the agents in case the project does not get provisioned. The refund bonus scheme is directly proportional to 
agent's contribution. Let $\mathcal{I}_{X}$ be an indicator random variable which takes the value 1 if $X$ is true and 0 otherwise. Then the utility structure of PPR, for each agent $i \in \mathbb{A}$ is given as,

$$
u_{i}(\cdot)=\mathcal{I}_{\mathcal{X} \geq h^{0}} \cdot\left(\theta_{i}-x_{i}\right)+\mathcal{I}_{\mathcal{X}<h^{0}} \cdot\left(\frac{x_{i}}{\mathcal{X}}\right) B,
$$

where $B$ is the total budget kept aside by the PM, and is distributed to the agents who contributed along with their contribution, as a refund, in case the project is not provisioned.

The refund bonus in PPR is independent of time of contribution and therefore all agents delay their contributions as close to the deadline as possible and wait to free-ride till the end. This may lead to the project not getting provisioned in practice (for eg., because of server failure near the deadline or transactions not getting processed in time). Therefore, such strategies are undesirable.

\section{Provision Point Mechanism with Securities (PPS)}

PPS addresses the shortcomings of PPR by offering agents refunds based on the time of their contribution. An early contributor is paid higher refund than a late contributor for the same contribution. The utility structure of PPS, for each agent $i \in \mathbb{A}$ is given as,

$$
u_{i}(\cdot)=\mathcal{I}_{\mathcal{X} \geq h^{0}} \cdot\left(\theta_{i}-x_{i}\right)+\mathcal{I}_{\mathcal{X}<h^{0}} \cdot\left(r_{i}^{t_{i}}-x_{i}\right),
$$

where, $t_{i}$ and $r_{i}^{t_{i}}$ are Agent $i$ 's time of contribution and the number of securities allocated to it, respectively. $r_{i}^{t_{i}}$ depends on its contribution $x_{i}$ at $t_{i}$, as well as the total number of securities issued in the market at time $t_{i}$, denoted by $q^{t_{i}}$, i.e. [Chandra et al., 2016, Eq. 6],

$$
r_{i}^{t_{i}}=C_{0}^{-1}\left(x_{i}+C_{0}\left(q^{t_{i}}\right)\right)-q^{t_{i}} .
$$

Here, $C_{0}$ is the cost function governing the underlying prediction market in PPS obtained from the general cost function $C$, by fixing the number of positive outcome securities. A cost function must satisfy [Chandra et al., 2016, CONDITIONS 1-4,6] to be used in PPS. The properties of the cost function, $C_{0}$, relevant to this paper are,

- Property 1. $r_{i}^{t_{i}}$ is a monotonically increasing function of $x_{i}$, i.e., $\frac{\partial r_{i}^{t_{i}}}{x_{i}}>1, \forall \theta_{i}<h^{0}, \forall i$ [Chandra et al., 2016, CONDITION-7].

- Property 2. The refund $r^{t_{i}}-x_{i}$ is a decreasing function of $t_{i}$ and $q^{t_{i}}$ as $q^{t_{i}}$ is non-decreasing function of $t_{i}, \forall i$ [Chandra et al., 2016, Step-2 (Theorem 3)].

The existing literature for civic crowdfunding is limited through its assumptions on the information structure of the contributing agents. We define an agent's information structure as consisting of its valuation and its belief towards the project's provision. Based on their valuations, we categorize agents as follows: positive (negative) agent i.e., Agent $i$ with $\theta_{i} \geq 0\left(\theta_{i}<0\right)$ or with positive (negative) preference towards $P P$ 's provision. Let $\mathbb{P}(\mathbb{N})$ denote the set of all positive (negative) agents, such that $\mathbb{A}=\mathbb{P} \cup \mathbb{N}$. Further, let $\vartheta^{1}=\sum_{i} \theta_{i} \forall i \in \mathbb{P}$ as the total valuation for $P P$ getting provisioned and $\vartheta^{2}=\sum_{i}\left(-\theta_{i}\right) \forall i \in \mathbb{N}$ as the total valuation for $P P$ not getting provisioned, i.e., $\vartheta=\vartheta^{1}-\vartheta^{2}$. For preference aggregation, the PM's goal is to determine whether the majority prefers $P P$ to be provisioned or not, i.e., whether or not $\vartheta \geq 0$.

We also consider agents with asymmetric beliefs towards $P P$ 's provision i.e., agents may believe that the project may be provisioned with probability (belief) $1 / 2 \pm \epsilon$ or may not be provisioned with probability $1 / 2 \mp \epsilon$ for some $\epsilon \geq 0$. Let $k_{i}^{1}=\left(1 / 2+\epsilon_{i}\right)$ and $k_{i}^{2}=\left(1 / 2-\epsilon_{i}\right)$ for some $\epsilon_{i} \geq 0$ such that $k_{i}^{1}+k_{i}^{2}=1, \forall i \in \mathbb{A}$. Let $\mathbb{A}^{+}\left(\mathbb{A}^{-}\right)$be the set of agents which believe that $P P$ will (will not) be provisioned i.e., every agent $i \in \mathbb{A}^{+}\left(i \in \mathbb{A}^{-}\right)$has belief $k_{i}^{1}\left(k_{i}^{2}\right)$ that $P P$ will be provisioned, such that $\mathbb{A}=\mathbb{A}^{+} \cup \mathbb{A}^{-}$.

In this paper, we require each agent to truthfully elicit its belief regarding the provision of the public project. Since an agent's opinion (belief) is its private information, we look for mechanisms which incentivize it to elicit its true opinion. In mechanism design theory, such mechanisms are called incentive compatible (IC). Further, these mechanisms must also be individually rational (IR) i.e., each Agent $i$ must have nonnegative utility ${ }^{1}$. Towards this, we make use of peer prediction mechanisms.

\subsection{Peer Prediction Mechanisms}

Peer prediction mechanisms (PPM) allow for elicitation and aggregation of subjective opinions from a set of agents. These are generally deployed in situations where there is no method of verifying an agent's honesty (of their opinion) or their ability. In the literature, there are a number of existing peer prediction mechanisms [Miller et al., 2005; Jurca and Faltings, 2007; Lambert and Shoham, 2008; Dasgupta and Ghosh, 2013; Radanovic and Faltings, 2013].

For illustrative purposes, in this paper, we focus on Robust Bayesian Truth Serum (RBTS) mechanism ([Witkowski and Parkes, 2012]).

\section{Robust Bayesian Truth Serum}

While RBTS mechanism properties hold for an arbitrary number of signals, we present the binary version of the mechanism, as we are interested in the elicitation of an agent's belief towards the provision of the project. In RBTS, each agent is required to submit, from [Witkowski and Parkes, 2012]:

1. Information Report: Let $f_{i}=\{0,1\}$ be Agent $i$ 's reported signal.

2. Prediction Report: Let $g_{i} \in[0,1]$ be Agent $i$ 's report about the frequency of high signals among the citizens.

Based on information report and prediction report, RBTS assigns a score to each agent. We describe the calculation of the scores in the complete version [Damle et al., 2019]. RBTS mechanism is IC and ex-post IR ([Witkowski and Parkes, 2012, Theorem 10]) for the elicitation of binary information for all $n \geq 3$, without relying on knowledge of the common prior. Note that many other peer prediction mechanisms are IC only when $n \rightarrow \infty$ and hence we chose RBTS.

${ }^{1}$ For more details on mechanism design, refer to [Garg et al., 2008a; Garg et al., 2008b] 


\section{Civic Crowdfunding for Agents With Negative Valuations}

In this section, we introduce a methodology through which civic crowdfunding mechanisms can incorporate symmetric agents with negative preferences (valuations) towards the public project's provision. For this, the PM sets up two separate markets, i.e., one for the provision and one against the provision of the project. Thus, agents now have a greater scope for manipulation. In such a setting, a strategic agent may choose to contribute in a market, against its preference, if its expected utility for contributing in that market is more than if it contributes in the market based on its preference. Therefore, to incorporate agents with negative preference, we must ingeniously construct the refund scheme in a way so that the agents are incentivized to contribute in the market based on their true preferences.

To illustrate this methodology, we provide two mechanisms for the same by adopting existing mechanisms in PPR and PPS; as namely, PPRN and PPSN. For these mechanisms, let $h^{1}\left(h^{2}\right)$ be the target for provision (rejection) of $P P$ with $\mathcal{X}^{1}\left(\mathcal{X}^{2}\right)$ as the total funding received towards (against) its provision. Further, let $h_{t}^{1}\left(h_{t}^{2}\right)$ denote the amount remaining for the project to be provisioned (not provisioned) at time $t$.

We present Provision Point Mechanism with Refund for Negative Preference (PPRN) leveraging PPR in the complete version of this paper [Damle et al., 2019]. In the next subsection, we present Provision Point Mechanism for Securities with Negative Preference (PPSN) by leveraging PPS.

\subsection{Provision Point Mechanism for Securities with Negative Preference (PPSN)}

We now propose a mechanism for civic crowdfunding for agents with negative valuations by leveraging PPS, namely PPSN.

\section{Protocol}

In PPSN, we consider a mechanism with two independent PPS prediction markets $-\mathrm{PPS}^{+}$and $\mathrm{PPS}^{-}$. In $\mathrm{PPS}^{+}$, agents contribute for the project to be provisioned (and buy negative securities) while in PPS $^{-}$, agents contribute for the project to not be provisioned (and buy positive securities). Note that the markets being independent, the prices in both the markets are also independent of the other. Provision point for $\mathrm{PPS}^{+}$is reached when the total contribution in it reaches $h^{1}$, and rejection point for PPS $^{-}$when the total contribution in it reaches $h^{2}$. Let, $\mathcal{X}^{1}$ be the total contribution received by the project in $\mathrm{PPS}^{+}$and $\mathcal{X}^{2}$ be the total contribution received by the project in $\mathrm{PPS}^{-}$. The goal of the mechanism is to provision the project or not based on whichever target is first reached.

Let $s_{i} \in\{1,2\}$, be a private preference variable for Agent $i$, such that $s_{i}=1, \forall i \in \mathbb{P}$ and $s_{i}=2, \forall i \in \mathbb{N}$.

\section{Common Refund Scheme}

An agent may not contribute in the market based on its preference if its expected refund is more in case it contributes in the other market. To prevent this, we present a common refund scheme that ensures that the agent obtains same refund in spite of which market it chooses to contribute. In this,
Agent $i$ contributes $x_{i}$ in any market based on a refund that depends on the minimum of the issued securities present in both the markets i.e., $Q^{t_{i}}=\min \left(q_{P P S^{+}}^{t_{i}}, q_{P P S^{-}}^{t_{i}}\right)$. Based on this, Agent $i$ is issued securities $\left(R_{i}^{t_{i}}\right)$ for a contribution $x_{i}$ given by

$$
R_{i}^{t_{i}}=C_{0}^{-1}\left(x_{i}+C_{0}\left(Q^{t_{i}}\right)\right)-Q^{t_{i}},
$$

from [Chandra et al., 2016, Eq. 6]. Thus, Agent $i$ 's refund in this scheme is $R_{i}^{t_{i}}-x_{i}$.

However, the number of issued securities only changes for the market in which the agent contributes $x_{i}$. For instance, if Agent $i$ contributes $x_{i}$ at time $t$ to $\mathrm{PPS}^{+}$, then the change in the issued securities in $\mathrm{PPS}^{+}$will be given by

$$
C_{0}^{-1}\left(x_{i}+C_{0}\left(q_{P P S^{+}}^{t}\right)\right)-q_{P P S^{+}}^{t} .
$$

Proposition 1. The securities allotted to an agent with total issued securities as $Q^{t}$ is always greater than or equal to those it would have received with securities $q_{P P S^{+}}^{t}$ or $q_{P P S^{-}}^{t}$ for the same contribution and the same cost function $C_{0}$.

Proof. The statement follows from the fact that the number of securities allotted, for the same contribution, is a decreasing function of the total issued securities (Property 2).

Proposition 2. The refund given by $R_{i}^{t_{i}}-x_{i}$ for Agent $i$, is a decreasing function with respect to time $t_{i}$.

Proof. The securities allotted to Agent $i, R_{i}^{t_{i}}$, decreases as $Q^{t_{i}}$ increases (Proposition 1). Further, since $Q^{t}=\min \left(q_{P P S^{+}}^{t}, q_{P P S^{-}}^{t}\right)$ and $q_{P P S^{+}}^{t}$ and $q_{P P S^{-}}^{t}$ are nondecreasing with respect to time $t$ (Property 2 ); $Q^{t}$ is a nondecreasing function of time. Thus, $R_{i}^{t_{i}}-x_{i}$ for Agent $i$, is a decreasing function with respect to time $t_{i}$.

Let us call $\mathrm{PPS}^{+}$as $p_{1}$, and $\mathrm{PPS}^{-}$as $p_{2}$. Thus, $p_{s_{i}}=$ $p_{1}, \forall i \in \mathbb{P}$ and $p_{s_{i}}=p_{2}, \forall i \in \mathbb{N}$. Further, let the market in which Agent $i$ contributes be $\tilde{p}_{s_{i}}$.

\section{Agent Utility}

The utility for Agent $i \in \mathbb{A}$ with $\tilde{p}_{s_{i}}=p_{1}$, in PPSN is as follows,

$$
u_{i}(\cdot)=I_{\mathcal{X}^{1} \geq h^{1}} \cdot\left(\theta_{i}-x_{i}\right)+I_{\mathcal{X}^{1}<h^{1}} \cdot\left(R_{i}-x_{i}\right)
$$

The utility for Agent $i \in \mathbb{A}$ with $\tilde{p}_{s_{i}}=p_{2}$, in PPSN is as follows,

$$
u_{i}(\cdot)=I_{\mathcal{X}^{2} \geq h^{2}} \cdot\left(-x_{i}\right)+I_{\mathcal{X}^{2}<h^{2}} \cdot\left(\theta_{i}+R_{i}-x_{i}\right)
$$

\section{Discussion.}

1. Trivially, from Eqs. 1 and 2, PPSN is (ex-post) IR. PPSN, however, is not IC [Damle et al., 2019].

2. The utility structure in PPSN can be intuitively explained as follows: As $\theta_{i}$ is Agent $i$ 's valuation for the project getting provisioned, it is accounted for when the project is provisioned, i.e., when $\mathcal{X}^{1} \geq h^{1}$ and $\mathcal{X}^{2}<h^{2}$. Further, in $\mathrm{PPS}^{+}$, the refund share is distributed when the provision point is not reached, i.e., when $\mathcal{X}^{1}<h^{1}$. Likewise, in PPS $^{-}$, the refund share is distributed when the rejection point is not reached, i.e., when $\mathcal{X}^{2}<h^{2}$. 


\section{Equilibrium Analysis}

Theorem 3.1 provides the equilibrium analysis of PPSN.

Theorem 3.1. For PPSN, with the utility as given by Eq. 1 and Eq. $2 \forall i \in \mathbb{A}, C: \mathbf{R}^{2} \rightarrow \mathbf{R}$ as the cost function, $C_{0}^{1}:$ $\mathbf{R} \rightarrow \mathbf{R}$ as the cost function obtained from $C$ by fixing the number positive outcome securities satisfying Property 1 and used in the market $p_{1}$ satisfying $\left(C_{0}^{1}\right)^{-1}\left(h^{1}+C_{0}(0)\right)<\vartheta^{1}$, and $C_{0}^{2}: \mathbf{R} \rightarrow \mathbf{R}$ as the cost function obtained from $C$ by fixing the number of negative outcome securities satisfying ${ }^{2}$ Property 1 and used in the market $p_{2}$ satisfying $C_{0}^{-1}\left(h^{2}+\right.$ $\left.C_{0}(0)\right)<\vartheta^{2}$, with $\vartheta^{1}>h^{1}$ and $\vartheta^{2}>h^{2}$, a set of strategies in the set $\sigma_{i}^{*}=\left(x_{i}^{*}, t_{i}^{*}, \tilde{p}_{s_{i}}\right)$,

$$
\sigma_{i}^{*}= \begin{cases}\left(0, a_{i}, p_{s_{i}}\right) & \text { if } \exists \text { l s.t. } h_{a_{i}}^{l}=0, \text { else, } \\ \left(x_{i}^{*}, a_{i}, p_{s_{i}}\right): & x_{i}^{*} \leq C_{0}\left(\left|\theta_{i}\right|+Q^{a_{i}}\right)-C_{0}\left(Q^{a_{i}}\right)\end{cases}
$$

$\forall l \in\{1,2\}$; are sub-game perfect equilibria $\forall i \in \mathbb{A}$, such that at equilibrium either $\mathcal{X}^{1}=h^{1}$ or $\mathcal{X}^{2}=h^{2}$ holds.

Proof. Since every agent $i \in \mathbb{A}$ is symmetric in its belief towards the project's provision, its expected utility is given by $1 / 2\left(\theta_{i}+R_{i}\right)-x_{i}$ irrespective of whether $\tilde{p}_{s_{i}}=p_{1}$ or $p_{2}$. Therefore, no agent has any incentive to deviate from its true preference. Hence, $\tilde{p}_{s_{i}}=p_{s_{i}}, \forall i \in \mathbb{A}$..

The rest of the proof follows similar to [Chandra et al., 2016] and is presented in [Damle et al., 2019].

Discussion. For PPSN, it can be seen that $\vartheta=\vartheta^{1}-\vartheta^{2}$. The project is always provisioned if $\vartheta^{1}>h^{1}$ and $\vartheta \geq 0$ or is never provisioned if $\vartheta^{2}>h^{2}$ and $\vartheta<0$. Here it must be noted that it can happen that $\vartheta^{1}>h^{1}$ and $\vartheta^{2}>h^{2}$ are simultaneously satisfied. In that case, if $\vartheta^{1}>\vartheta^{2}$, the project attains provision point faster than rejection point and vice-versa.

The significance of this result is that, in PPSN (and PPRN), at equilibrium, the project is provisioned if the majority prefers it, i.e., only when $\vartheta \geq 0$. Thus, this methodology allows for truthful aggregation of private preference of each agent with respect to public projects.

\section{Civic Crowdfunding for Agents with Asymmetric Beliefs}

In this section, we present a General Mechanism which incentivizes agents with asymmetric beliefs towards the public project's provision, to contribute towards it. In this section, we restrict our attention to the case where every agent has a positive valuation towards project's provision.

The General Mechanism involves two phases: a Belief Phase (BP) and a Contribution Phase (CP). In BP, each Agent $i$ submits its belief for the provision of the project for which it is allocated some share (denoted by $b_{i}$ ) of the reward calculated through Belief Based Reward (BBR) scheme described in the next subsection. In CP, each Agent $i$ submits its contribution $\left(x_{i}\right)$ to the project which is dependent on the refund obtained in the BP as well as on the provision point mechanism deployed for civic crowdfunding.

\footnotetext{
${ }^{2}$ Trivially, both the cost functions are the same. Hence, from hereon we refer to both of them without the superscript i.e., as $C_{0}$.
}

The mechanism requires two separate bonuses for both the phases, which the PM announces at the start of the project. Let $B^{B}\left(B^{C}\right)$ be the bonus allocated for the BP and the CP, respectively. Further, let $a_{i}^{1}\left(a_{i}^{2}\right)$ be the time at which Agent $i$ arrives to the mechanism for the BP (CP) with $t_{i}^{1}\left(t_{i}^{2}\right)$ as the time at which it reports its belief (contribution). Let the deadline for the BP (CP) be $T^{B}\left(T^{C}\right)$ announced at the start.

Unlike in the case of civic crowdfunding for agents with symmetric beliefs, an asymmetric agent which has significant belief towards the project getting provisioned or not, may choose to free-ride and not contribute. Therefore, we introduce a reward scheme that further incentivizes such agent's to contribute towards the project.

\subsection{Belief Based Reward (BBR)}

To quantitatively measure the reward share to be distributed to every contributing agent in the BP, we use a PPM. We consider PPMs which incentivize truthful elicitation of an agent's belief i.e., PPMs which are IC.

Let the score of Agent $i$ depending on its belief $(1 / 2 \pm$ $\left.\epsilon_{i}\right)$ be $m_{i}$. Further, let $S^{t_{i}}$ be the set consisting of all the agents that have reported their belief including the Agent $i$, who reports its belief at time $t_{i}$. For $T^{B}$ as the deadline, $S^{T^{B}}$ consists of all the agents that have reported their belief. Let $m_{i}, \forall i \in S^{T^{B}}$ be the agent scores calculated after the deadline. For,

$$
w_{i}=\frac{m_{i}}{\sum_{j} m_{j}} \forall j \in S^{t_{i}},
$$

Agent $i$ 's reward in the scheme is,

$$
b_{i}=\left\{\begin{array}{lll}
\frac{w_{i}}{\sum_{j} w_{j}} \times B^{B} & \forall j \in \mathbb{A}^{+} ; \quad \forall i \in \mathbb{A}^{+} \\
\frac{w_{i}}{\sum_{j} w_{j}} \times B^{B} & \forall j \in \mathbb{A}^{-} ; \quad \forall i \in \mathbb{A}^{-}
\end{array}\right.
$$

We refer to the reward scheme given by Eq. 3 as Belief Based Reward (BBR). With this, we show the following proposition:

Proposition 3. BBR is a decreasing function of time.

Proof. From Eq. 3, BBR is inversely proportional to the order in which agents report their beliefs. As the arrival of agents is non-decreasing w.r.t. time, $\mathrm{BBR}$ is a decreasing function of time.

In addition, BBR is also strongly budget balanced, i.e., in BBR the entire budget is utilized.

\section{RBTS Reward Scheme}

In this paper, we use RBTS Mechanism to calculate the mechanism score $m_{i}$ for each Agent $i$. For this, every agent submits its prediction and information report as described earlier.

In this reward scheme, let $f_{i}=0$ denote that Agent $i$ has belief that the project will be provisioned and $f_{i}=1$ denote that Agent $i$ has belief that the project will not be provisioned. Thus, through each agent's prediction report, the PM knows whether an agent belongs to the set $\mathbb{A}^{+}$or the set $\mathbb{A}^{-}$.

We present Provision Point Mechanism with Refunds for Agents with Asymmetric Beliefs (PPRx) by plugging PPR refund bonus scheme for the CP in [Damle et al., 2019]. In the next subsection, we present Provision Point Mechanism with Securities for Agents with Asymmetric Beliefs (PPSx) by plugging PPS refund bonus scheme for the CP. 


\subsection{Provision Point Mechanism with Securities for Agents with Asymmetric Beliefs (PPSx)}

In PPSx, we plugin PPS refund bonus scheme for the CP.

\section{Agent Utility}

The utility for Agent $i \in \mathbb{A}^{+}$, in PPSx is as follows,

$$
u_{i}(\cdot)=I_{\mathcal{X} \geq h^{0}} \cdot\left(\theta_{i}-x_{i}+b_{i}\right)+I_{\mathcal{X}<h^{0}} \cdot\left(r_{i}-x_{i}\right)
$$

Similarly, the utility for Agent $i \in \mathbb{A}^{-}$, in PPSx is as follows,

$$
u_{i}(\cdot)=I_{\mathcal{X} \geq h^{0}} \cdot\left(\theta_{i}-x_{i}\right)+I_{\mathcal{X}<h^{0}} \cdot\left(r_{i}-x_{i}+b_{i}\right)
$$

\section{Discussion.}

1. From Eqs. 4 and 5, PPSx is (ex-post) IR. PPSx, however, is not IC [Damle et al., 2019].

2. At the end of the mechanism, only one set of agents, either $\mathbb{A}^{+}$or $\mathbb{A}^{-}$, get the BBR reward.

\section{Equilibrium Analysis}

Theorem 4.1 presents the equilibrium analysis of PPSx. We formally prove the theorem in [Damle et al., 2019].

Theorem 4.1. For PPSx, with the utility as given by Eq. 4 and Eq. 5, $\forall i \in \mathbb{A}^{+}, C: \mathbf{R}^{2} \rightarrow \mathbf{R}$ as the cost function, with $C_{0}: \mathbf{R} \rightarrow \mathbf{R}$ as the cost function obtained from $C$ by fixing the number positive outcome securities satisfying Property 1 , $C_{0}^{-1}\left(h^{0}+C_{0}(0)\right)<\vartheta$ with $B^{B}, B^{C}>0$ and Belief Phase reward calculated as per Eq. $3 \forall i \in \mathbb{A}$, a set of strategies $\sigma_{i}^{*}=\left(x_{i}^{*}, t_{i}^{1 *}, t_{i}^{2 *}\right)$ in the set,

$\sigma_{i}^{*}=\left\{\begin{array}{l}\left(0, a_{i}^{1}, a_{i}^{2}\right) ; \text { if } h_{a_{i}^{2}}^{0}=0, \text { else } \\ \left(x_{i}^{*}, a_{i}^{1}, a_{i}^{2}\right): x_{i}^{*} \leq C_{0}\left(\theta_{i}+b_{i}+q^{a_{i}^{2}}\right)-C_{0}\left(q^{a_{i}^{2}}\right),\end{array}\right.$

$\forall i \in \mathbb{A}^{+}$and a set of strategies $\sigma_{i}^{*}=\left(x_{i}^{*}, t_{i}^{1 *}, t_{i}^{2 *}\right)$ in the set,

$\sigma_{i}^{*}=\left\{\begin{array}{l}\left(0, a_{i}^{1}, a_{i}^{2}\right) ; \text { if } h_{a_{i}^{2}}^{0}=0, \text { else, } \\ \left(x_{i}^{*}, a_{i}^{1}, a_{i}^{2}\right): x_{i}^{*} \leq C_{0}\left(\theta_{i}-b_{i}+q^{a_{i}^{2}}\right)-C_{0}\left(q^{a_{i}^{2}}\right),\end{array}\right.$

$\forall i \in \mathbb{A}^{-}$; are sub-game perfect equilibria such that at equilibrium $\mathcal{X}=h^{0}$ holds.

Discussion. Trivially, for PPSx, the upper bound for the equilibrium contributions $\forall i \in \mathbb{A}^{+}$(with $k_{i}^{1}$ as the belief towards project's provision) is greater than the upper bound for the equilibrium contributions $\forall i \in \mathbb{A}^{-}$(with $k_{i}^{2}$ as the belief towards project's provision; $k_{i}^{1}+k_{i}^{2}=1$ ), for the same $\theta_{i}$ and $b_{i}$. This implies that agents with greater belief towards the project's provision contribute more than agents with lesser belief towards it. Thus, BBR and the utility structure as given by Eqs. 4 and 5 for PPSx, provides a natural way for civic crowdfunding with asymmetric agents such that the project is provisioned at equilibrium. We now discuss the setting of up the markets for these mechanisms.

\section{Discussion}

For PPSN (PPRN), the PM is required to set up two independent PPS (PPR) markets. The provision point for these projects are determined based on the economics of their construction. The rejection point can be similarly determined. For instance, the rejection point for our garbage dump yard example could be the cost of constructing the dump yard at a different locality. Another method for determining the rejection point could be the cost incurred by the government as a result of the public project not getting provisioned. An instance of this could be the construction of dams. The cost of not setting up the dam, i.e., the rejection point for the project could be the cost incurred by the government in providing electricity or water etc. to the nearby areas which they could have achieved through the dam's construction. Note that, the amount collected if the project is rejected is at the discretion of the government.

The PM should allocate reasonable budget for all these mechanisms. Allocating huge budgets may not guarantee the provision/rejection of the projects. In such a case, the agents may prefer to contribute just enough to get substantial refunds. Likewise, allocating insignificant budgets may prove to not be incentivising enough for agents to contribute to the market. Further, in PPSN, the cost function, $C_{0}$, used to allocate the securities must also be same for both markets. Additional details for setting up the prediction markets as well as the budget can be found in [Chandra et al., 2016; Chandra et al., 2017].

Mechanisms for Asymmetric Agents with Negative Valuations. Civic crowdfunding mechanisms for agents with information structure consisting of both - their preferences and their belief towards the provision of the project is still illusive as in such a setting, agents have an extra dimension to manipulate the mechanism. For instance, combining PPSN and PPSx (PPRN and PPRx) will not suffice. As formally shown in [Damle et al., 2019], an Agent $i \in \mathbb{A}^{+}$with $\theta_{i} \geq 0$, will always choose to contribute towards the project not getting provisioned, as it believes that the project will be provisioned anyways, making it eligible for the additional refund bonus. Likewise, an Agent $i \in \mathbb{A}^{-}$with $\theta_{i}<0$ will always contribute towards the provision of the project. However, an Agent $i \in \mathbb{A}^{+}$with $\theta_{i}<0$ and an Agent $i \in \mathbb{A}^{-}$with $\theta_{i} \geq 0$ will always contribute as per its true preference.

Thus, the general method and the general mechanism proposed in this paper for civic crowdfunding for agents with negative valuations and agents with asymmetric beliefs respectively, are not sufficient to incentivize every asymmetric agent to contribute as per their true preferences.

\section{Conclusion}

In this paper, we explored the limitations of existing literature on civic crowdfunding. We showed that it poses restrictions on the information structure of agents, as it only allows for positive as well as symmetric agents. We broke this barrier on the information structure of an agent by proposing (i) a general methodology for addressing symmetric agents with negative preferences based on which we proposed two mechanisms, PPRN and PPSN (Theorem 3.1); and (ii) a general mechanism for positive agents with asymmetric beliefs based on which we proposed two mechanisms, PPRx and PPSx (Theorem 4.1). We leave it for future work to explore the feasibility of combining negative preferences and asymmetric belief into one framework for civic crowdfunding. 


\section{References}

[Alaei et al., 2016] Saeed Alaei, Azarakhsh Malekian, and Mohamed Mostagir. A dynamic model of crowdfunding. In Proceedings of the 2016 ACM Conference on Economics and Computation, EC '16, pages 363-363, New York, NY, USA, 2016. ACM.

[Arieli et al., 2017] Itai Arieli, Moran Koren, and Rann Smorodinsky. The crowdfunding game. In Proceedings of the 13th International Conference on Web and Internet Economics, WINE 2017, 2017.

[Bagnoli and Lipman, 1989] Mark Bagnoli and Barton L Lipman. Provision of public goods: Fully implementing the core through private contributions. The Review of Economic Studies, 56(4):583-601, 1989.

[Brubaker, 1975] Earl R Brubaker. Free ride, free revelation, or golden rule? The Journal of Law and Economics, 18(1):147-161, 1975.

[Chandra et al., 2016] Praphul Chandra, Sujit Gujar, and Y Narahari. Crowdfunding public projects with provision point: A prediction market approach. In ECAI, pages 778786, 2016.

[Chandra et al., 2017] Praphul Chandra, Sujit Gujar, and Yadati Narahari. Referral-embedded provision point mechanisms for crowdfunding of public projects. In Proceedings of the 16th Conference on Autonomous Agents and MultiAgent Systems AAMAS 2018, pages 642-650. International Foundation for Autonomous Agents and Multiagent Systems, 2017.

[Chen, 2008] Yan Chen. Incentive-compatible mechanisms for pure public goods: A survey of experimental research. Handbook of experimental economics results, 1:625-643, 2008.

[Damle et al., 2018] Sankarshan Damle, Moin Hussain Moti, Sujit Gujar, and Praphul Chandra. Designing refund bonus schemes for provision point mechanism in civic crowdfunding. arXiv preprint arXiv: 1810.11695, 2018.

[Damle et al., 2019] Sankarshan Damle, Moin Hussain Moti, Praphul Chandra, and Sujit Gujar. Civic crowdfunding for agents with negative valuations and agents with asymmetric beliefs. arXiv preprint arXiv:1905.11324, 2019.

[Dasgupta and Ghosh, 2013] Anirban Dasgupta and Arpita Ghosh. Crowdsourced judgement elicitation with endogenous proficiency. In Proceedings of the 22nd international conference on World Wide Web, pages 319-330. ACM, 2013.

[Garg et al., 2008a] Dinesh Garg, Y Narahari, and Sujit Gujar. Foundations of mechanism design: A tutorial part 1-key concepts and classical results. Sadhana, 33(2):83, 2008.

[Garg et al., 2008b] Dinesh Garg, Y Narahari, and Sujit Gujar. Foundations of mechanism design: A tutorial part 2advanced concepts and results. Sadhana, 33(2):131, 2008.
[Groves and Ledyard, 1977] Theodore Groves and John Ledyard. Optimal allocation of public goods: A solution to the" free rider" problem. Econometrica: Journal of the Econometric Society, pages 783-809, 1977.

[Jurca and Faltings, 2007] Radu Jurca and Boi Faltings. Robust incentive-compatible feedback payments. In AgentMediated Electronic Commerce. Automated Negotiation and Strategy Design for Electronic Markets, pages 204218. Springer, 2007.

[Lambert and Shoham, 2008] Nicolas Lambert and Yoav Shoham. Truthful surveys. In International Workshop on Internet and Network Economics, pages 154-165. Springer, 2008.

[Miller et al., 2005] Nolan Miller, Paul Resnick, and Richard Zeckhauser. Eliciting informative feedback: The peerprediction method. Management Science, 51(9):13591373, 2005.

[Radanovic and Faltings, 2013] Goran Radanovic and Boi Faltings. A robust bayesian truth serum for non-binary signals. In Proceedings of the 27th AAAI Conference on Artificial Intelligence (AAAI" 13), pages 833-839, 2013.

[Schmidtz, 1991] David Schmidtz. The limits of government (boulder), 1991.

[Shen et al., 2018] Wen Shen, Jacob W Crandall, Ke Yan, and Cristina V Lopes. Information design in crowdfunding under thresholding policies. In Proceedings of the 17th International Conference on Autonomous Agents and MultiAgent Systems AAMAS 2018, pages 632-640. International Foundation for Autonomous Agents and Multiagent Systems, 2018.

[Strausz, 2017] Roland Strausz. A theory of crowdfunding: A mechanism design approach with demand uncertainty and moral hazard. American Economic Review, 107(6):1430-76, 2017.

[Tabarrok, 1998] Alexander Tabarrok. The private provision of public goods via dominant assurance contracts. Public Choice, 96(3-4):345-362, 1998.

[Witkowski and Parkes, 2012] Jens Witkowski and David C Parkes. A robust bayesian truth serum for small populations. In $A A A I$, volume 12, pages 1492-1498, 2012.

[Zubrickas, 2014] Robertas Zubrickas. The provision point mechanism with refund bonuses. Journal of Public Economics, 120:231-234, 2014. 